\title{
Interaction of a Spatial Soliton on an Interface between Two Nonlinear Media
}

\author{
Mohd Azid Mat Din ${ }^{1^{*}}$, Bakhram Umarov², Nor Amirah Busul Aklan³ and Aizuddin Ahmad Kamely 4 \\ 1,3 Department of Computational and Theoretical Sciences, Faculty of Science, International Islamic \\ University Malaysia, 25200 Kuantan, Malaysia \\ 2,4 Department of Physics, Faculty of Science, International Islamic University Malaysia, 25200 Kuantan, \\ Malaysia
}

Spatial solitons are the solutions of nonlinear partial differential equations describing the propagation of optical beams in nonlinear medium. This paper studies the scattering of a spatial solitons of the Cubic-Quintic Nonlinear Schrödinger Equation (C-Q NLSE) on an interface between two nonlinear media. The scattering process will be investigated by variational approximation method and by direct numerical solution of C-Q NLSE. This variational approximation method has been used to analyse the dynamic of the width and centre of mass position of a soliton during the scattering process. Meanwhile, a direct numerical simulation of C-Q NLSE was done to check the accuracy of the approximation by using the same range of parameters and initial condition. The results for direct numerical simulation of CQNLSE for soliton parameters are quite similar with the variational equation. The studies showed that soliton can be reflected by or transmitted through the interface, also the nonlinear surface wave can be formed depending on the parameters of interface and initial soliton.

Keywords: Spatial Soliton; Cubic-Quintic Nonlinear Schrodinger Equation; Soliton Scattering; Variational Methods; Nonlinear Surface Wave

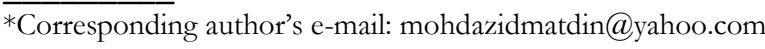




\section{INTRODUCTION}

In optics, the term solitons can be considered as a localized wave propagating in a nonlinear media with constant speed and shape (Porsezian \& Kuriakose, 2003; Abdullaev et al.,2014). A pulse or a beam have a natural tendency to spread, either due to chromatic dispersion or because of spatial diffraction. But under some condition's nonlinearity may compensate natural broadening and a stable selflocalized wave packet may be formed (Ablowitz \& Clarkson,1991; Yang, 2010). These kinds of objects are known as an optical soliton (Kivshar \& Agrawal,2003). The optical beams that propagate in a nonlinear medium without diffraction is called spatial optical soliton (Trillo \& Torruellas, 2013; Chen et al., 2012). Beam radius stays invariant during propagation. One can imagine that a spatial soliton is a result of an exact compensation between diffraction and nonlinearly induced self-focusing effects. Also, one can consider it as an optical beam inducing a waveguide which directs itself while propagating as if it were bounded in the long tube.

The principle on how an optical beam can guide itself in self-induced waveguide was first introduced by Askaryan (1962) and soon the self-guiding or self-focusing phenomena was observed in experiment (Hercher, 1964). Later, the spatial optical solitons were observed in experiment both in 2 dimensional (Barthelemy et al., 1985). and 3 dimensional cases (Bjorkholm \& Ashkin, 1974).

The study of spatial solitons represents interest for fundamental physics of nonlinear waves but also there had been proposals for some interesting applications, for example wave guiding and beam splitting, optical interconnects, frequency conversion, image transmission, gateless computing and soliton based navigation (Trillo \& Torruellas, 2013; Chen et al., 2012). The experimental and theoretical studies of spatial solitons reveal that they may show the particle-like behaviour, they interact between each other and with external potentials preserving their identity.

To control and manage spatial solitons it is necessary to study their interaction with different types of perturbations. Especially important problem is to learn how the optical spatial solitons behave on the interface between two media with different optical properties. This problem was first studied theoretically in the papers (Aceves et al., 1989;Aceves et al., 1988) where a spatial soliton in Kerr nonlinear medium were described as a soliton solution of nonlinear Schrödinger equation (NLSE) and the equivalent particle theory for soliton was developed. It was shown that the success of this theory depends on the ability of the spatial solitons to remain unaffected by perturbations which allowed us to describe the interaction of spatial solitons with interface as a soliton with slowly modulated parameters. An analytical expression for nonlinear of GoosHänchen shift has been derived and the equivalent potential was obtained. The studies showed that soliton can be reflected by or transmitted through the interface, also the nonlinear surface wave can be formed depending on the parameters of interface and initial soliton. It was demonstrated that the analytical estimation provides good agreement with the direct numerical simulation of NLSE.

It has been widely known that the optical beam can selffocus in both space and time while propagating in a nonlinear media. However, this beam also can be collapse in a Kerr medium for 2 and 3 dimensional when it produces high electric field strengths. It was observed that the insertion of a saturable nonlinearity could prevent the singular collapse, thus it is necessary to consider nonlinearities higher than third order (Biswas\&Konar,2006). Therefore, in this paper we will try to understand the propagation of optical solitons in nonKerr law media which induces a fifth order nonlinearity term. The problem we are going to consider is to study the interaction of a spatial soliton with interface between two nonlinear media, when both cubic and quintic nonlinearity will be considered. The spatial soliton in this case will be represented as a soliton solution of generalized C-Q NLSE.

The plan of the present paper is as follows. In Sec II we briefly describe the model and the main equation for scattering of spatial soliton with nonlinear interface and obtain the perturbed CQ-NLSE. Sec III we present variational approximation method and illustrate the equivalent potential. We then describe in Sec IV two representative set of numerical result for ordinary differential equation (ODE) and reveal the kinds of soliton transmission, reflection and trapping behaviours. In Sec V 
we make comparison between the partial differential equation (PDE) and ODE result by using same range of parameter. The paper is concluded in the Sec VI.

\section{THE MODEL AND MAIN EQUATIONS}

The main equation of our model is based on the C-Q NLSE of known as Gross-Pitaevskii equation (GPE) with perturbation

$$
i \psi_{t}+\frac{1}{2} \psi_{x x}+\alpha|\psi|^{2} \psi+\beta|\psi|^{4} \psi=V_{1}(x) \psi-V_{2}(x)|\psi|^{2}
$$

where $\Psi(x, t)$ is the wave function, $\alpha$ and $\beta$ are the coefficients of cubic and quintic nonlinearity respectively. The perturbation potentials $V_{1}(x)$ and $V_{2}(x)$ are

$$
\begin{array}{r}
V_{1}(x)=g_{1} H(x) \\
V_{2}(x)=g_{2} H(x) \\
H(x)= \begin{cases}0, & x<0 \\
1, & x \geq 0\end{cases}
\end{array}
$$

Here $\mathrm{H}(\mathrm{x})$ is a step function and characterized by the strengths $g_{1}>0$ and $g_{2}>0$. We consider positive coefficient of nonlinearity, $\alpha>O$ and $\beta>O$ corresponds to focusing nonlinearity on optic applications so that the system support bright solitons. In the case $V(x)=O$ and $\alpha=1$, eq. (1) has exact solution in the form of C-Q NLSE soliton

$$
\begin{gathered}
\psi(x, t)=\frac{A_{1}}{\left[1+a_{1} \cosh \left[B_{1}(x-\xi(t)]^{\frac{1}{2}}\right.\right.} \exp (i \theta) \\
B_{1}=\sqrt{2} A_{1}, \quad a_{1}=\sqrt{1+\frac{4}{3} \beta A_{1}^{2}}, \quad \xi=v, \quad k=-v \\
\omega=\sqrt{\left(\frac{A_{1}^{2}}{4}\right)-\left(\frac{k^{2}}{2}\right)} \\
\theta(x, t)=-k x+w t+\varphi
\end{gathered}
$$

where $A_{1}, \xi, v, \varphi$ are the amplitude, center of mass position, velocity and initial phase of the soliton respectively.

\section{VARIATIONAL APPROXIMATION METHOD}

Variational approximation is a body of deterministic methods for making approximate inference for parameters in complex models. The capability of this approximation to reduce the partial differential equation to ordinary equation and be a well described analysis of non-integrable equation has made it a widely used theoretical tool in the research of solitons. Moreover, it also shows agreement with the numerical simulation results in many cases where the very first application was for soliton in plasma physics.

The variational approximation can be used to obtain the dynamic properties of the solution of NLSE. The accuracy of this approximation critically depends on the chosen of our trial function. In this paper, a Lagrangian density was derived first from the C-Q NLSE before substituted the trial function into the equation

$$
\begin{aligned}
& \mathrm{L}=\frac{i}{2}\left(\psi \psi_{t}^{*}-\psi^{*} \psi_{t}\right)+\frac{1}{2}\left|\psi_{x}\right|^{2}-\frac{\beta}{3}|\psi|^{2} \\
& -\frac{1}{2}\left|\psi_{x}\right|^{4}+g_{1} H(x)|\psi|^{2}-\frac{1}{2} g_{2} H(x)|\psi|^{4}
\end{aligned}
$$

As next step, we find the averaged/effective Lagrangian with spatial integration of lagrangian density $L=\int_{-\infty}^{\infty} \mathcal{L} d x$ using the trial function (Aklan\& Umarov,2015)

$$
\psi(x, t)=A \operatorname{sech}\left(\frac{x-\xi}{a}\right) \mathrm{e}^{i b(x-\xi)^{2}+i v(x-\xi)+i \varphi}
$$

where $A, a, v, \xi, \mathrm{b}$ and $\varphi$ represent amplitude, width, velocity, centre of mass position, chirp parameter, and phase of the soliton, respectively. It gives rise to the total averaged Lagrangian in eq. (7). The conserved quantity $N=$ $\int_{-\infty}^{+\infty}|\Psi|^{2} d x=a A^{2} \sqrt{\pi}$ is the norm of wave function, where the number of atom, $N$ is constant for every trajectory of the system.

$$
\begin{aligned}
L= & N\left[\frac{\pi^{2} a^{2} b_{t}}{12}+\frac{\pi^{2} a^{2} b^{2}}{6}-\frac{\xi_{t}}{2}+\varphi_{t}+\frac{1}{6 a^{2}}-\frac{N}{6 a}+\right. \\
& \frac{g_{1}}{2}\left(1-\tanh \left(-\frac{\xi}{a}\right)\right)+\frac{g_{2} N}{24 a} \tanh \left(-\frac{\xi}{a}\right) \operatorname{sech}^{2}\left(-\frac{\xi}{a}\right) \\
& \left.-\frac{g_{2} N}{12 a}\left(1-\tanh \left(-\frac{\xi}{a}\right)\right)-\frac{2 N^{2} \beta}{45 a^{2}}\right]
\end{aligned}
$$

The variation approximation equation is derived through Euler-Lagrange equation $d / d t\left(\partial L / \partial q_{t}\right)-(\partial L / \partial q)$ where $q$ are parameters of wave function.

$$
\begin{aligned}
& \frac{d}{d t} \frac{\partial L}{\partial a_{t}}-\frac{\partial L}{\partial a}=0, \frac{d}{d t} \frac{\partial L}{\partial b_{t}}-\frac{\partial L}{\partial b}=0, \\
& \frac{d}{d t} \frac{\partial L}{\partial \xi_{t}}-\frac{\partial L}{\partial \xi}=0, \frac{d}{d t} \frac{\partial L}{\partial \varphi_{t}}-\frac{\partial L}{\partial \varphi}=0,
\end{aligned}
$$


From above equation, we get a set of coupled equations for width and centre-of-mass position of soliton

$$
\begin{aligned}
a_{t t}= & \frac{4}{\pi^{2} a^{3}}-\frac{2 N}{\pi^{2} a^{2}}+\frac{6 g_{1} \xi}{\pi^{2} a^{2}} \operatorname{sech}^{2}\left(-\frac{\xi}{a}\right)+ \\
& \frac{g_{2} N}{2 \pi^{2} a^{2}} \tanh \left(-\frac{\xi}{a}\right) \operatorname{sech}^{2}\left(-\frac{\xi}{a}\right)-\frac{g_{2} N \xi}{2 \pi^{2} a^{3}} \operatorname{sech}^{4}\left(-\frac{\xi}{a}\right)+ \\
& \frac{g_{2} N \xi}{\pi^{2} a^{3}} \tanh ^{2}\left(-\frac{\xi}{a}\right) \operatorname{sech}^{2}\left(-\frac{\xi}{a}\right)-\frac{g_{2} N}{\pi^{2} a^{2}}\left(1-\tanh \left(-\frac{\xi}{a}\right)\right) \\
& \frac{g_{2} N \xi}{2 \pi^{2} a^{3}} \operatorname{sech}^{2}\left(-\frac{\xi}{a}\right)-\frac{16 N^{2} \beta}{15 \pi^{2} a^{3}} \\
\xi_{t t}= & -\frac{g_{1}}{2 a} \operatorname{sech}^{2}\left(-\frac{\xi}{a}\right)+\frac{g_{2} N}{24 a^{2}} \operatorname{sech}^{4}\left(-\frac{\xi}{a}\right)- \\
& \frac{g_{2} N}{12 a^{2}} \tanh ^{2}\left(-\frac{\xi}{a}\right) \operatorname{sech}^{2}\left(-\frac{\xi}{a}\right)+\frac{g_{2} N}{12 a^{2}} \operatorname{sech}^{2}\left(-\frac{\xi}{a}\right)
\end{aligned}
$$

Equation (9) and (10) represent the main result of this work and it describe the scattering of soliton on interface between two nonlinear media. The system become coupled when the soliton come close to the interface and we should consider for full system of eq. (9) - (10). In this case, the soliton is transmitted or reflected through the interface and it also will generate small oscillation due to the perturbation.

But when the soliton is located far from the potential $\xi>>a$, it is clear from the equations above are decoupled and one has a soliton moving freely with initial velocity and its amplitude also remain constant. In this case, we can neglect the effect of potential to the width of soliton, hence it is possible to find the approximation width of the stationary soliton $(a t t=O)$ of cubic-quintic NLSE.

$$
a_{s}=\sqrt{\frac{15}{A^{2}\left(15+16 A^{2} \beta\right)}}
$$

Thus, we can consider the eq. (9) - (10) are separately for each other and can be transform into a single equation

$$
\begin{aligned}
\xi_{t t} & =-\frac{d V_{p}(\xi)}{d \xi}=\frac{g_{1}}{2 a_{s}} \operatorname{sech}^{2}\left(-\frac{\xi}{a_{s}}\right)-\frac{g_{2} N}{24 a_{s}^{2}} \operatorname{sech}^{4}\left(-\frac{\xi}{a_{s}}\right) \\
& +\frac{g_{2} N}{12 a_{s}^{2}} \tanh ^{2}\left(-\frac{\xi}{a_{s}}\right) \operatorname{sech}^{2}\left(-\frac{\xi}{a_{s}}\right)-\frac{g_{2} N}{12 a_{s}^{2}} \operatorname{sech}^{2}\left(-\frac{\xi}{a_{s}}\right)
\end{aligned}
$$

The above equation then can be integrated once and reduced to eq. (13) which is that for a classical particle motion in the external potential, $V_{p}$ in eq. (14).

$$
\begin{gathered}
\xi_{t}=\sqrt{2 V_{p}(\xi)} \\
V_{p}=-\frac{g_{1}}{2} \tanh \left(-\frac{\xi}{a_{s}}\right)+\frac{g_{2} N}{8 a_{s}} \tanh \left(-\frac{\xi}{a_{s}}\right)-\frac{g_{2} N}{24 a_{s}} \tanh ^{3}\left(-\frac{\xi}{a_{s}}\right)
\end{gathered}
$$

the values of $g_{2}$ in eq. (14). In Figure 1, it shows that if $g_{2}<$ 0.42 , there are no critical value for $V_{p}$ since there are no corresponding steady state solution at left side of medium [13]. Differently, if $g_{2}>0.42$, there exist a maximum point in $V_{p}$ due to the present of an unstable steady state surface wave near the interface between two nonlinear media.

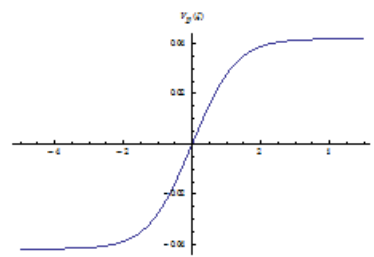

(a)

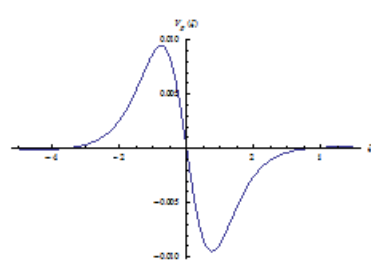

(c)

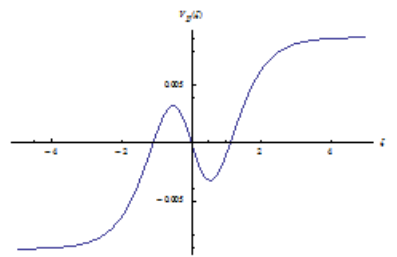

(b)

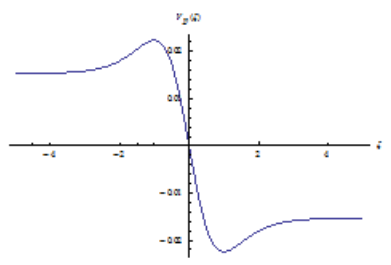

(d)
Figure 1. Graphs of the effective potential, $V_{p}$ at the interface $(x=O)$ for $g_{1}=0.1, A=0.7, \beta=1$ and various values of the parameter $g_{2}:$ (a) $g_{2}=0.1$, (b) $g_{2}=0.5$, (c) $g_{2}=0.61$, (d) $g_{2}=0.8$.

Now, we focus on the potential at right hand of the media. There are four typical cases to be observed: the first one refers to 1 (a) which is $g_{2}<0.42$, where $V_{p}$ is an increasing function of $x$. Three of others correspond to the cases where $g_{2}>0.42$ and it demonstrate a minimum point of $V_{p}$, but in 1(b) $V_{p}(\mathrm{o})<V_{p}(\infty), 1(\mathrm{c}) V_{p}(\mathrm{o})=V_{p}(\infty)$ and $1(\mathrm{~d})$ $V_{p}(0)>V_{p}(\infty)$. According to the cases, the critical point is due to the existences of a stable steady state solution in right side media. As a result, the soliton that approaching the interface from the right will be circle at the centre and return back to $+\infty$ without passing though the interface. This happen because of its initial energy less than $V_{p}(\mathrm{o})$. However, the soliton that propagate with enough energy will cross the interface.

Hence, we can conclude that here are exist a minimum and a maximum value of this potential when $0.42<g_{2}<$ 1.5. As this occurs, if $0.42<g_{2}<0.61$, then $V_{p}(\infty)>V_{p}$ (o) and if0.61 $<g_{2}<1.5$, then $V_{p}(\infty)<V_{p}(0)$.

\section{ODE SIMULATION OF THE INITIAL VALUE PROBLEM}

The different shapes of the potential are determined by 
We now describe a set of numerical result for the ODE which is the equation (9) - (10). We initialize the soliton at $\xi(0)=-8$ and $\mathrm{v}(O)=v$ with $v>O$, propagating rightward toward the interface. To set it propagate from the right side, we fixed the values of $\xi(0)=8$ and $v(o)=v$ with $v<O$ while the soliton amplitude is set to $\mathrm{A}=0.7$ and $g_{1}=0.1$.

In Figure 2, the behaviour for $g_{2}=0.8$ is shown under 4 trajectory types; (1) a soliton coming from the left and failing to pass through the interface, therefore ending up at $\xi=-\infty$; (2) a soliton entering from the left and ending up at $\xi=\infty$ because it has enough energy to overcome $V_{\max }$; (3) a packet coming from the right with enough energy to overcome $V_{\max }$ and it pass through the interface; (4) a packet moving from $\xi=\infty$ and it reflected from the interface.

When the soliton entering from the left medium, the critical velocity of the soliton, $V_{c}=0.118$ while from the right it given that $\quad V_{c}=-0.339$. The value of $\mid V_{c}$, right $\mid>$ $\mid V_{c}$, left $\mid$ due to the energy needed to overcome the potential are higher when the soliton entering from right compared to the left. The above condition only will be occurred if $V_{p}(\infty)<V_{p}(0)$ where $g_{2}$ should be greater than 0.61 .
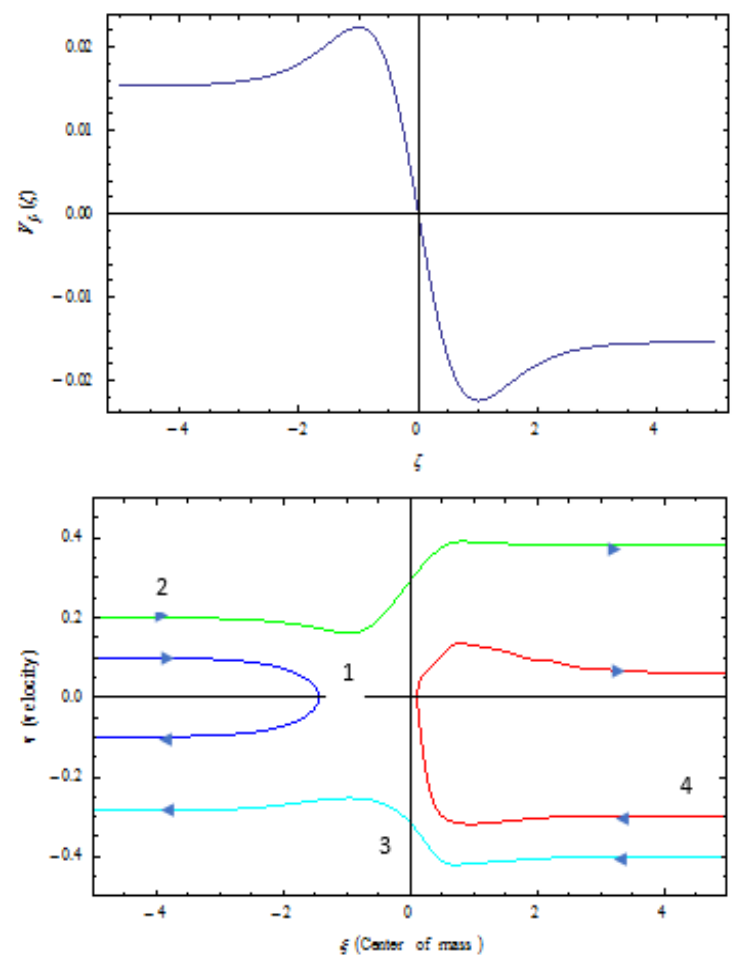

Figure 2. Potential and phase plane for values of the parameter $g_{1}=0.1$ and $g_{2}=0.8$.
In Figure 3, we computed ODE for $g_{2}=0.5$. The result is quite similar compared to previous cases in Figure 2; when the soliton has enough energy to overcome $V_{\max }$, it will be transmitted through the interface (case (2) and case (3)) and will be reflected by the interface when the soliton is in low energy (case (1)). But in case (4), the soliton is trapped for a while at the interface and then it moves out from the potential well to the right side of the medium. The escape of the soliton from the potential well is due to the action of resonance force that causes the destruction of the surface wave (Abdullaev et al., 1998). The formation of the nonlinear surface wave occurs when the soliton is captured into the minimum of the potential well. It will be oscillating around the potential minimum under the effect of a periodic force. When the amplitude of oscillation exceeds the critical value of the modulation amplitude, hence it can leave the interface.
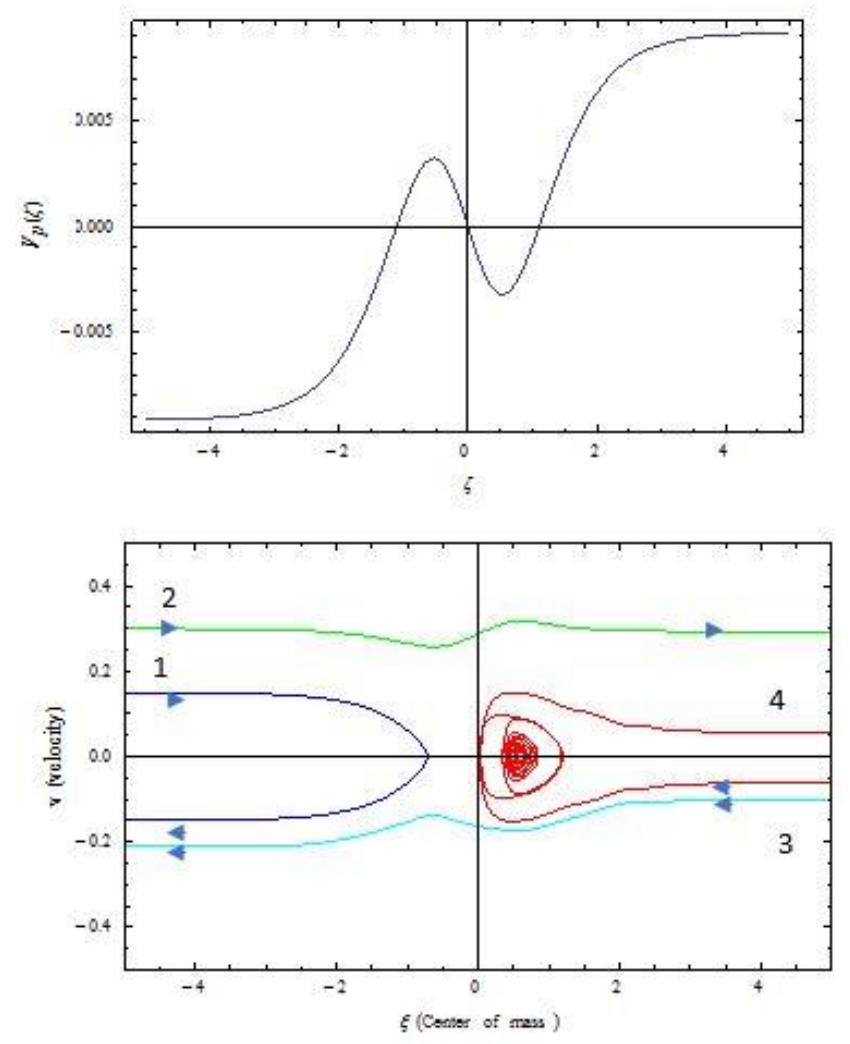

Figure 3. Potential and phase plane for values of the parameter $g_{1}=0.1$ and $g_{2}=0.5$.

\section{NUMERICAL RESULT}

In order to study the dynamics of solitons, split-step Fast Fourier Transform (FFT) procedure is applied to solve the C-Q NLSE numerically(Aklan \& Umarov, 2015); in our 
case we use a first-order split step method as our numerical scheme. The time step was taken as 0.001 and 2048 number of FFT mode were used. The spatial domain was taken as $[-12 \pi, 12 \pi]$. We applied absorbing boundary layers at both ends of the integration domain to minimize the radiation that can feed back into the system. To reduce numerical error, we operated our simulation with wider spatial domain, larger grid points and smaller time steps.

The variational approximation method gave the approximate results and based on some assumptions. The direct numerical solutions of the governing equations are performed to check the accuracy of the approximate calculations by monitoring the evolution of centre-of mass position of the soliton by using eq. (15) and compared with dynamic equation of variational approximation Eq. (9) $-(10)$.

$$
\xi(t)=\frac{\int_{-\infty}^{\infty} x|\psi(x, t)|^{2} d x}{\int_{-\infty}^{\infty}|\psi(x, t)|^{2} d x}
$$

In Figure 4 the interaction of soliton can be observed through the time dependence of its relative centre-ofmass trajectory. The soliton at initial width, $a(0)=a_{s}$ and position $\xi_{0}=8$ is set in motion with velocity $v$, toward an interface between two nonlinear media. In Figure 4 we present the reflected, transmitted, and trapped part of the soliton as a function of the incident velocity. As we can see from the Figure, the soliton moves freely with constant width and velocity when it far from an interface, but it will be affected when it approaches an interface.

According to the results obtained from numerical simulation, it is quite good agreement between the result of PDE (dotted blue line) and ODE (red line). Though, there is still a slightly different between the ODE model and the PDE model due to the omission of energy radiation in the ODE model. This radiation mostly generated when a spatial soliton passes through the interface. Because of this radiation loss, we observed that each time it crosses the new medium it will be reshaped and no longer be a perfect soliton. This is the reason why the translation motion (gradient of the centre of mass) of soliton in the PDE model is slightly lower than the ODE model after crossing. Since the energy losses are quite small, we find that a good agreement between the result of PDE and ODE. It shows that the variational analysis is well describes a soliton scattering process in cubic and quintic nonlinearity.

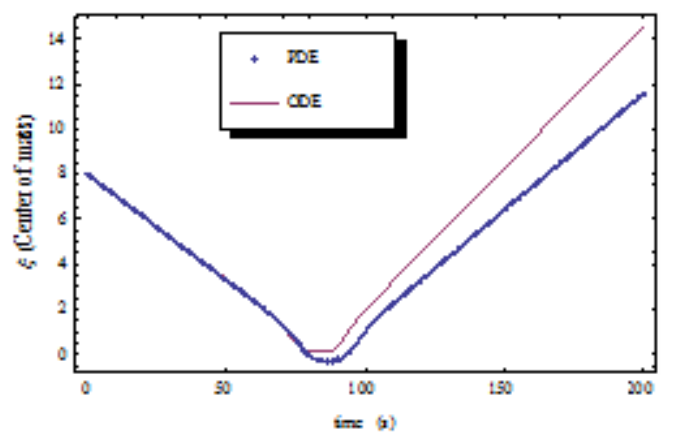

(a)

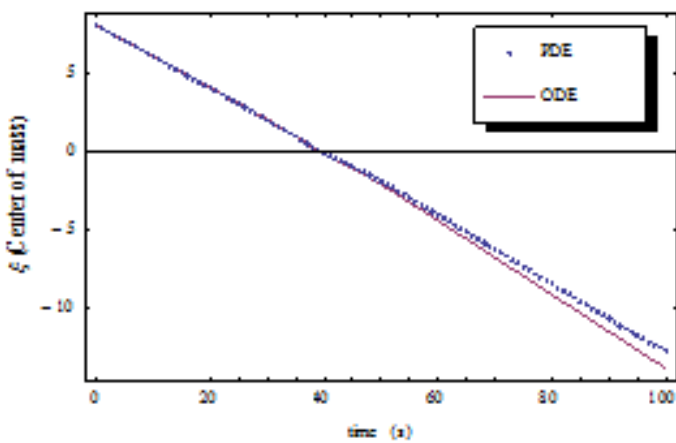

(b)

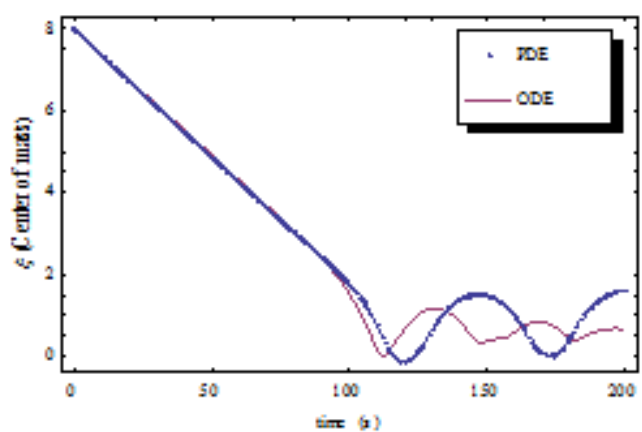

(c)

Figure 4. Evolution of the centre-of-mass position of a soliton with $\mathrm{A}=0.7, \mathrm{~A}_{1}=1.14, g_{1}=0.1, g_{2}=0.5, \xi_{0}=8$ and velocity as variable, $v$ : (a) $v=-0.093$, (b) $v=-0.2$, (c) $v=-$ 0.0615 .

\section{CONCLUSION}

We have studied the scattering of solitons of NLSE with cubic and quintic terms added to the system in the presence of two potential $V_{1}(x)$ and $V_{2}(x)$. The results of C-Q NLSE numerical simulation show that variational approximation 
well described the soliton scattering on interface between two nonlinear media with the consideration of a good choice of trial function. Direct simulation of C-Q NLSE has already covered all the results needed but can be time consuming to run the simulation, thus variational approach can show the results faster and also it gives us the insight to the physics of the soliton scattering. We hope that this simulation will help us to develop intuition and to solve more complicated problems on soliton scattering. This researched can be extended on soliton interactions with localized potential walls and wells and also other form of generalized NLSE. This result will serve as guidelines for possible future experiments with matter-wave solitons and optical solitons.

\section{ACKNOWLEDGEMENT}

The author would like to thank to research grant No. FRGS16-014-0513 of the Ministry of Higher Education and IRAGS18-039-0040 of International Islamic University Malaysia.

\section{REFERENCES}

Abdullaev, F. K., Baizakov, B. B., \& Umarov, B. A. (1998). Resonance phenomena in interaction of a spatial soliton with the modulated interface of two nonlinear media. Optics communications, 156(4-6), 341-346.

Abdullaev, F., Darmanyan, S., Khabibullaev, P., \& Engelbrecht, J. (2014). Optical solitons. Springer Publishing Company, Incorporated.

Ablowitz, M. J., \& Clarkson, P. A. (1991). Solitons, nonlinear evolution equations and inverse scattering (Vol. 149). Cambridge university press.

Aceves, A. B., Moloney, J. V., \& Newell, A. C. (1988). Reflection and transmission of self-focused channels at nonlinear dielectric interfaces. Optics letters, 13(11), 1002-1004.

Aceves, A. B., Moloney, J. V., \& Newell, A. C. (1989). Theory of light-beam propagation at nonlinear interfaces. I. Equivalent-particle theory for a single interface. Physical Review A, 39(4), 1809.

Aklan, N. A. B., \&Umarov, B. (2015). Interaction of solitons with delta potential in the Cubic-Quintic Nonlinear Schrodinger Equation. (ICREM7) (pp. 93-96). IEEE.

Aklan, N. A. B., \& Umarov, B. (2015, October). The soliton scattering of the cubic-quintic nonlinear Schrödinger equation on the external potentials.
In AIP Conference Proceedings (Vol. 1682, No. 1, p. 020022). AIP Publishing.

Askaryan, G. A. (1962). Effect of the gradient of a strong electromagnetic ray on electrons and atoms. Zhur. Eksptl'. iTeoret. Fiz., 42.

Barthelemy, A., Maneuf, S., \& Froehly, C. (1985). Propagation soliton et auto-confinement de faisceaux laser par non linearitéoptique de Kerr. Optics communications, 55(3), 201-206.

Biswas, A., \& Konar, S. (2006). Introduction to nonKerr law optical solitons. Chapman and Hall/CRC.

Bjorkholm, J. E., \& Ashkin, A. A. (1974). CW selffocusing and self-trapping of light in sodium vapor. Physical Review Letters, 32(4), 129.

Chen, Z., Segev, M., \& Christodoulides, D. N. (2012). Optical spatial solitons: historical overview and recent advances. Reports on Progress in Physics, 75(8), 086401.

Hercher, M. (1964). Laser-induced damage in transparent media. J. Opt. Soc. America, 54, 563.

Kivshar, Y. S., \& Agrawal, G. (2003). Optical solitons: from fibers to photonic crystals. Academic press.

Porsezian, K., \& Kuriakose, V. C. (Eds.). (2003). Optical solitons: theoretical and experimental 
ASM Science Journal, Volume 13, 2020

challenges (Vol. 613). Springer Science \& Business Media.

Trillo, S., \& Torruellas, W. (Eds.). (2013). Spatial solitons (Vol. 82). Springer.

Yang, J. (2010). Nonlinear waves in integrable and nonintegrable systems (Vol. 16). Siam. 